\title{
Synthesis, Characterization and X-Ray Crystal Structures of Cyclam Derivatives. 8. A Thermodynamic and Kinetic Appraisal of Lead(II) Chelation by Octadentate Carbamoyl-Armed Macrocycles
}

François Cuenot, Michel Meyer, Enrique Espinosa, and Roger Guilard*

Laboratoire d'Ingénierie Moléculaire pour la Séparation et les Applications des Gaz (LIMSAG, UMR 5633 du CNRS), Université de Bourgogne, Faculté des Sciences, 6 boulevard Gabriel, 21100 Dijon, France.

Supporting Information for

Manuscript $N^{\circ}$ IC 0508019

* Author to whom correspondence should be addressed.

E-mail: Roger.Guilard@u-bourgogne.fr. Phone: (33) 3803961 11. Fax: (33) 380396117. 


\section{Content}

Figure S1 $\quad{ }^{1} \mathrm{H}-{ }^{1} \mathrm{H}$ NMR COSY spectrum of $\left[\mathrm{Pb}\left(\mathbf{L}^{2}\right)\right]\left(\mathrm{ClO}_{4}\right)_{2} \bullet 0.5 \mathrm{H}_{2} \mathrm{O}$ in DMSO- $d_{6}$.

Table S1 Pseudo-first order rate constants $\left(k_{\mathrm{obs}}\right)$ for the complexation of lead(II) by ligands $\mathbf{L}^{\mathbf{1}}, \mathbf{L}^{\mathbf{2}}$, and $\mathbf{L}^{\mathbf{3}}$.

Table S2 Pseudo-first order rate constants $\left(k_{\mathrm{obs}}\right)$ for the proton-assisted dissociation of the lead(II) complexes $\mathrm{Pb}\left(\mathbf{L}^{\mathbf{1}}\right)^{2+}, \operatorname{Pb}\left(\mathbf{L}^{\mathbf{2}}\right)^{2+}$, and $\mathrm{Pb}\left(\mathbf{L}^{\mathbf{3}}\right)^{2+}$. 


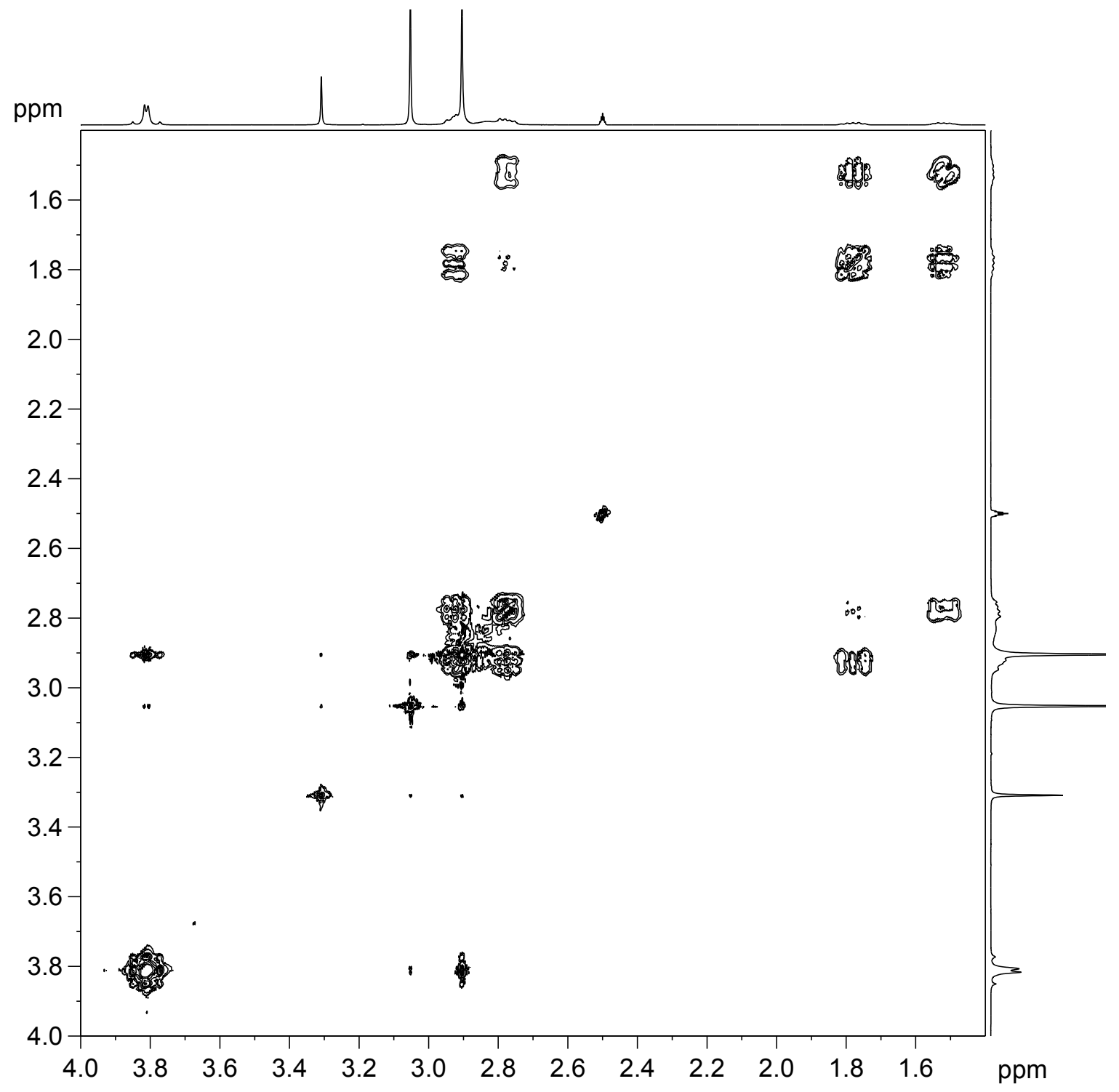

Figure S1. $\quad{ }^{1} \mathrm{H}-{ }^{1} \mathrm{H}$ NMR COSY spectrum of $\left[\mathrm{Pb}\left(\mathbf{L}^{2}\right)\right]\left(\mathrm{ClO}_{4}\right)_{2} \bullet 0.5 \mathrm{H}_{2} \mathrm{O}$ in DMSO- $d_{6} . T=$ $300 \mathrm{~K} ; 500 \mathrm{MHz}$. 
Table S1. Pseudo-first order rate constants $\left(k_{\mathrm{obs}}\right)$ for the complexation of lead(II) by ligands $\mathbf{L}^{1}, \mathbf{L}^{2}$, and $\mathbf{L}^{3 a}$

\begin{tabular}{|c|c|c|c|c|c|c|c|c|}
\hline $\mathrm{p}[\mathrm{H}]$ & $\begin{array}{l}{\left[\mathbf{L}^{1}\right]_{\mathrm{tot}} \times 10^{4}} \\
(\mathrm{M})\end{array}$ & $\begin{array}{l}k_{\mathrm{obs}} \\
\left(\mathrm{s}^{-1}\right)\end{array}$ & $\mathrm{p}[\mathrm{H}]$ & $\begin{array}{l}{\left[\mathbf{L}^{2}\right]_{\text {tot }} \times 10^{4}} \\
(\mathrm{M})\end{array}$ & $\begin{array}{l}k_{\mathrm{obs}} \\
\left(\mathrm{s}^{-1}\right)\end{array}$ & $\mathrm{p}[\mathrm{H}]$ & $\begin{array}{l}{\left[\mathbf{L}^{3}\right]_{\mathrm{tot}} \times 10^{4}} \\
(\mathrm{M})\end{array}$ & $\begin{array}{l}k_{\mathrm{obs}} \\
\left(\mathrm{s}^{-1}\right)\end{array}$ \\
\hline \multirow[t]{5}{*}{4.29} & 2.06 & $6.391(5)$ & \multirow[t]{5}{*}{5.08} & 2.10 & $2.225(2)$ & \multirow[t]{5}{*}{5.09} & 2 & $1.989(2)$ \\
\hline & 3.91 & $8.027(5)$ & & 3.91 & 3.893(3) & & 3.93 & $3.552(3)$ \\
\hline & 5.96 & $9.855(6)$ & & 5.90 & $5.758(4)$ & & 4.89 & 4.397(3) \\
\hline & 7.85 & $11.526(6)$ & & 7.81 & $7.548(5)$ & & 6.86 & $6.208(4)$ \\
\hline & 9.67 & $13.039(9)$ & & 9.60 & $9.076(7)$ & & 8.77 & $8.033(5)$ \\
\hline \multirow[t]{5}{*}{4.45} & 2.06 & $6.226(4)$ & \multirow[t]{5}{*}{5.40} & 2.11 & $4.268(4)$ & \multirow[t]{5}{*}{5.40} & 2.09 & $3.775(3)$ \\
\hline & 3.91 & $8.535(5)$ & & 3.91 & $7.805(6)$ & & 4 & $7.336(6)$ \\
\hline & 5.96 & $11.316(5)$ & & 5.91 & $11.847(9)$ & & 6.15 & $11.026(8)$ \\
\hline & 7.85 & $13.823(9)$ & & 7.80 & $15.49(1)$ & & 7.88 & $14.75(1)$ \\
\hline & 9.67 & $16.35(1)$ & & 9.60 & 18.81(2) & & 9.7 & $17.156(4)$ \\
\hline \multirow[t]{5}{*}{4.69} & 2.06 & $6.118(4)$ & \multirow[t]{5}{*}{5.71} & 2.11 & $8.408(9)$ & \multirow[t]{5}{*}{5.71} & 2.09 & $7.734(7)$ \\
\hline & 3.91 & $11.195(7)$ & & 3.91 & $15.75(1)$ & & 4 & $14.92(1)$ \\
\hline & 5.97 & $16.192(8)$ & & 5.91 & 24.12(2) & & 6.15 & $23.46(1)$ \\
\hline & 7.86 & $20.54(1)$ & & 7.80 & $31.60(2)$ & & 7.88 & $29.82(2)$ \\
\hline & 9.67 & $24.93(2)$ & & 9.60 & $39.03(4)$ & & 9.70 & $36.78(6)$ \\
\hline \multirow[t]{5}{*}{4.88} & 2.06 & $9.978(7)$ & \multirow[t]{5}{*}{5.93} & 2.11 & 13.91(1) & \multirow[t]{5}{*}{5.95} & 2.01 & $11.56(1)$ \\
\hline & 3.91 & $17.28(1)$ & & 3.91 & $26.20(2)$ & & 3.93 & $23.63(2)$ \\
\hline & 5.97 & $26.17(2)$ & & 5.91 & 40.29(3) & & 5.90 & $36.23(3)$ \\
\hline & 7.86 & $34.20(2)$ & & 7.81 & $52.69(5)$ & & 7.80 & 47.86(3) \\
\hline & 9.68 & $41.81(3)$ & & 9.60 & 64.74(7) & & 9.70 & $59.40(4)$ \\
\hline \multirow{5}{*}{5.09} & 2.06 & $15.79(1)$ & \multirow{5}{*}{6.14} & 2.11 & $23.24(3)$ & \multirow{5}{*}{6.14} & 2.01 & $19.06(2)$ \\
\hline & 3.91 & $28.73(2)$ & & 3.92 & 43.61(3) & & 3.93 & $38.18(3)$ \\
\hline & 5.97 & $44.90(3)$ & & 5.91 & $66.35(5)$ & & 5.90 & $58.08(4)$ \\
\hline & 7.71 & $57.53(4)$ & & 7.81 & $87.87(7)$ & & 7.80 & $76.99(5)$ \\
\hline & 9.68 & $72.09(4)$ & & 9.61 & $107.7(1)$ & & 9.70 & $96.34(7)$ \\
\hline \multirow[t]{5}{*}{5.48} & 2.06 & $33.35(3)$ & \multirow[t]{5}{*}{6.37} & 2.11 & $39.178(4)$ & \multirow[t]{5}{*}{6.39} & 2 & $31.33(3)$ \\
\hline & 3.91 & $61.56(5)$ & & 3.92 & $73.40(5)$ & & 3.93 & $64.75(4)$ \\
\hline & 5.98 & $98.19(7)$ & & 5.91 & 111.64(9) & & 5.90 & $98.89(7)$ \\
\hline & 7.87 & 131.1(1) & & 7.82 & $146.6(1)$ & & 7.85 & $132.38(9)$ \\
\hline & 9.70 & $158.8(2)$ & & 9.61 & 181.0(2) & & 9.70 & $164.4(2)$ \\
\hline \multirow[t]{5}{*}{5.85} & 2.06 & $69.37(7)$ & \multirow[t]{5}{*}{6.63} & 2.11 & 70.74(6) & \multirow[t]{5}{*}{6.64} & 2.02 & $57.51(6)$ \\
\hline & 4.08 & $124.7(2)$ & & 3.92 & $132.8(1)$ & & 3.94 & $115.50(8)$ \\
\hline & 5.99 & $207.2(3)$ & & 5.91 & 199.6(2) & & 5.92 & $174.1(2)$ \\
\hline & 7.89 & $277.6(5)$ & & 7.82 & 263.6(4) & & 7.84 & $230.5(3)$ \\
\hline & 9.72 & $336(2)$ & & 9.62 & $323.5(6)$ & & 9.75 & $288.7(4)$ \\
\hline \multirow[t]{10}{*}{6.05} & 2.06 & $113.98(9)$ & 6.79 & 2.12 & $91.2(1)$ & 6.78 & 2.09 & $82.92(6)$ \\
\hline & 3.92 & $207.2(3)$ & & 3.92 & $180.7(2)$ & & 4 & $160.9(1)$ \\
\hline & 5.99 & $326.3(7)$ & & 5.91 & 267.7(4) & & 6.16 & $245.4(2)$ \\
\hline & 7.90 & $423(2)$ & & 7.82 & $352.5(6)$ & & 7.9 & $311.8(5)$ \\
\hline & 9.74 & $505(2)$ & & 9.62 & $435(1)$ & & 9.72 & $395.1(9)$ \\
\hline & & & 6.88 & 2.12 & $114.4(1)$ & 6.96 & 2.01 & \\
\hline & & & & 3.93 & $213.5(2)$ & & 2.95 & \\
\hline & & & & 5.92 & $323.4(6)$ & & 3.94 & \\
\hline & & & & 7.82 & $423.7(9)$ & & 4.90 & \\
\hline & & & & 9.63 & 496(2) & & 6.89 & \\
\hline
\end{tabular}

${ }^{a}\left[\mathrm{~Pb}^{2+}\right]_{\text {tot }}=2.015 \times 10^{-5} \mathrm{M} ;[\mathrm{MES}]=0.025 \mathrm{M} ; I=0.1 \mathrm{M}(\mathrm{H}, \mathrm{Na}) \mathrm{ClO}_{4} ; T=298.2(2) \mathrm{K}$. 
Table S2. Pseudo-first order rate constants $\left(k_{\mathrm{obs}}\right)$ for the proton-assisted dissociation of the lead(II) complexes $\mathrm{Pb}\left(\mathbf{L}^{\mathbf{1}}\right)^{2+}, \operatorname{Pb}\left(\mathbf{L}^{\mathbf{2}}\right)^{2+}$, and $\mathrm{Pb}\left(\mathbf{L}^{\mathbf{3}}\right)^{2+a}$

\begin{tabular}{cccc}
\hline$\left[\mathrm{HClO}_{4}\right]_{\text {tot }} \times 10^{4}$ & \multicolumn{3}{c}{$k_{\text {obs }}\left(\mathrm{s}^{-1}\right)$} \\
\cline { 2 - 4 }$(\mathrm{M})$ & {$\left[\mathrm{Pb}\left(\mathbf{L}^{\mathbf{1}}\right)\right]^{2+}$} & {$\left[\mathrm{Pb}\left(\mathbf{L}^{2}\right)\right]^{2+}$} & {$\left[\mathrm{Pb}\left(\mathbf{L}^{3}\right)\right]^{2+}$} \\
\hline 2.07 & $11.79(2)$ & $3.537(5)$ & $1.2572(8)$ \\
5.18 & $32.30(4)$ & $10.57(1)$ & $3.926(4)$ \\
10.4 & $54.1(1)$ & $19.29(2)$ & $7.92(1)$ \\
15.5 & $70.5(1)$ & $26.54(3)$ & $11.82(2)$ \\
20.7 & $83.3(2)$ & $32.99(4)$ & $15.84(2)$ \\
25.9 & $95.0(1)$ & $38.61(5)$ & $19.87(3)$ \\
36.2 & $112.0(3)$ & $48.44(6)$ & $27.21(4)$ \\
51.8 & $135.7(2)$ & $61.19(8)$ & $38.35(8)$ \\
67.3 & $156.6(4)$ & $73.0(1)$ & $49.4(1)$ \\
81.5 & $170.9(5)$ & $82.5(1)$ & $59.1(2)$ \\
103.5 & $190.9(5)$ & $95.2(1)$ & $71.6(2)$ \\
155.3 & $235.9(5)$ & $122.9(2)$ & $100.5(3)$ \\
207 & $277.0(6)$ & $147.3(2)$ & $128.3(4)$ \\
258.8 & $308.1(8)$ & $170.5(3)$ & $150.9(5)$ \\
310.6 & $338.9(9)$ & $191.3(4)$ & $172.5(6)$ \\
362.3 & $375(1)$ & $209.6(4)$ & $190.3(7)$ \\
414.1 & $402(1)$ & $230.5(5)$ & $216(1)$ \\
465.8 & $422(1)$ & $248.6(5)$ & $239.2(9)$ \\
517.6 & $447(1)$ & $269.6(6)$ & $259(1)$ \\
\hline
\end{tabular}

${ }^{a}\left[\mathrm{~Pb}\left(\mathbf{L}^{\mathbf{1}}\right)^{2+}\right]_{\text {tot }}=1.84 \times 10^{-5} \mathrm{M} ;\left[\mathrm{Pb}\left(\mathbf{L}^{2}\right)^{2+}\right]_{\text {tot }}=1.80 \times 10^{-5} \mathrm{M} ;\left[\mathrm{Pb}\left(\mathbf{L}^{3}\right)^{2+}\right]_{\text {tot }}=1.13 \times 10^{-5} \mathrm{M} ; I=$ $0.1 \mathrm{M}(\mathrm{H}, \mathrm{Na}) \mathrm{ClO}_{4} ; T=298.2(2) \mathrm{K}$. 